\title{
Fluid-solid transition in unsteady shearing flows
}

\author{
Dalila Vescovi ${ }^{1, \star}$, Diego Berzi ${ }^{1}$, and Claudio di Prisco ${ }^{1}$ \\ ${ }^{1}$ Department of Civil and Environmental Engineering, Politecnico di Milano, 20133 Milano, Italy
}

\begin{abstract}
This paper focuses on the mechanical behaviour of granular systems under shearing, unsteady conditions. The results of numerical simulations of time evolving, homogeneous, shear flows of an assembly of frictional spheres, under constant volume conditions are illustrated. Simulations have been performed considering three volume fractions corresponding to fluid, solid and near-to-critical conditions at steady state. The three systems follow very different evolutionary paths, in terms of pressure, coordination number and stress ratio. Fluid-like and solid-like systems exhibit large and small fluctuations, respectively, in those quantities. A critical value of the coordination number seems to govern the transition from fluid to solid.
\end{abstract}

\section{Introduction}

The discontinuous and inhomogeneous nature of granular materials leads to complex mechanical behaviours, even in case of simple flow conditions. In particular, granular systems can behave like either fluids, meaning that they yield under shear stress, or like solids able to resist applied stresses without deforming [1]. If grains are widely spaced and free to move in any direction, interacting only through collisions, the medium behaves like a fluid and the stresses are proportional to the square of the strain rate under simple shearing (Bagnold scaling [2]). On the other hand, when particles are densely packed, a network of persistent contacts develops within the medium, and the granular material shows a solid-like, rate-independent behaviour. The mechanical response of the system during the solid-fluid transition, is still an open question, [3] although several constitutive models have been proposed to this purpose in the literature [4-9].

Whereas several numerical results have been obtained in the literature concerning steady, shearing granular flows, unsteady conditions have been less investigated. In this work, we investigate the fluid-solid transition in unsteady, homogeneous, shear flows of a collection of identical, frictional spheres.

\section{DEM numerical simulations}

The authors have performed DEM numerical simulations of unsteady simple shear flows of frictional spheres, using a linear spring-dashpot interaction model [10, 11], implemented in the open-source code Mercury-DPM (www.mercurydpm.org) [12, 13]. All the simulations have been carried out under constant volume in a cubic box of dimension $L$ (figure 1). 2000 particles of diameter

\footnotetext{
^e-mail: dalila.vescovi@polimi.it
}

$d$, density $\rho_{p}$, i.e., mass $m_{p}=\rho_{p} \pi d^{3} / 6$, were used. The size $L$ of the domain has been chosen once the volume fraction $v$ is fixed (where, for a granular material composed of identical particles, the local volume fraction $v$ is defined as the ratio of the local material density to the particle mass density). We set the normal coefficient of restitution $e_{n}=0.7$, the normal spring stiffness $k_{n}=10^{7} \mathrm{~Pa} \mathrm{~m}$, the tangential spring stiffness $k_{t}=2 / 7 k_{n}$ (corresponding to a tangential coefficient of restitution $\left.e_{t}=e_{n}=0.7\right)$ and the inter-particle friction coefficient $\mu=0.3$. In order to keep the system homogeneous in space, Lees-Edwards [14] periodic boundary conditions in the $z$ direction and periodic boundary conditions in the $x$ and $y$ directions have been employed. The system is sheared by moving the boundaries in opposite directions at constant horizontal velocity $V$, and setting the global shear rate $\hat{\gamma}=2 V / L$ equal to $0.1 \mathrm{~s}^{-1}$. The instantaneous application of a relative velocity between the boundaries is not immediately transmitted within the entire granular material, given that particles in the core of the domain cannot initially feel the boundaries motion. As a consequence, the velocity profiles are not linear, instead they exhibit some curvature; the local shear rate $\dot{\gamma}$ (velocity gradient) is not homogeneous, but is maximum at the boundaries and minimum (close to zero) in the core. After a short time interval $(0<t<0.005$ s) the non-homogeneity is dissipated and $\dot{\gamma}$ becomes homogeneous in space and almost constant in time, equal to the global shear rate $\hat{\dot{\gamma}}=0.1 \mathrm{~s}^{-1}$.

Three values of the constant volume fraction have been taken into consideration: $0.59,0.60$ and 0.62 . For steady, shearing flows, solid volume fraction larger (lower) than a critical, $v_{c}$, indicates that a granular system is solid-like (fluid-like), i.e., rate-independent components of the stresses are present (absent). $v_{c}$ is the largest volume fraction at which a randomly collisional granular material can be sheared without force chains spanning the entire 
domain [15]. The critical volume fraction depends on the granular material, in particular is affected by the polydispersity of the system [16, 17] and the inter-particle friction coefficient [1]. Chialvo et al. [6] have measured that for identical particles with $\mu=0.3, v_{c}$ is 0.598 . Then, our three samples at steady state should be in fluid $(v=0.59)$, solid $(v=0.62)$ and near-to-critical $(v=0.60)$ conditions. At steady state, there is a univocal relation between the critical volume fraction and the corresponding critical coordination number $C_{c}$ (where the coordination number $C$ is defined as the average number of contacts between all particles), independent of the shear rate [9]. The coordination number is an important parameter for describing the interaction among grains and the characteristic time scale of the force chains [18]. Note that in this paper we are not disregarding particles with zero (floaters) or one contact (rattlers) even if they do not participate to the contact network. The "corrected" coordination number $C^{*}$ has been introduced by neglecting floaters and rattlers $[17,19]$, and is related to the standard coordination number as $C=C^{*}\left(1-\phi_{r}\right)$ [20], where $\phi_{r}$ is the rattler fraction. In this work, we assume the system to experience a transition from fluid to solid states, under unsteady conditions, independently of the volume fraction, when the coordination number reaches its critical value $C_{c}$. The corrected coordination number at the transition, $C_{c}^{*}$, has been measured by Sun and Sundaresan [21], and, for $\mu=0.3, C_{c}^{*}=4.6$. By assuming $\phi_{r}=0.13 \pm 0.03$ as measured for frictionless particles [20], the critical coordination number is $C_{c} \approx 4$.

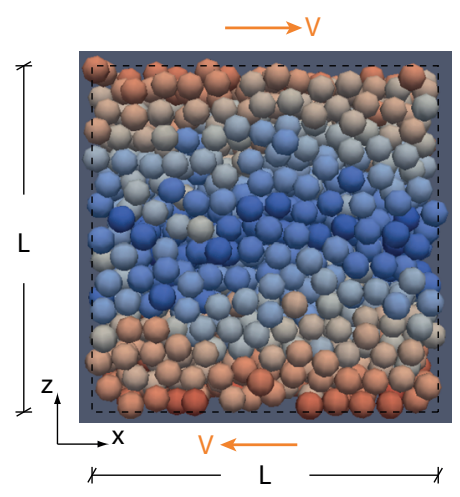

Figure 1. Sketch of the flow configuration. A granular material composed of identical, frictional spheres is homogeneously sheared at constant global shear rate $\hat{\dot{\gamma}}$, with flow in horizontal direction (boundaries move in opposite horizontal directions with velocity $V=\hat{\dot{\gamma}} L / 2$ ), by using Lees Edwards boundary conditions. Colours indicate speed, from blue (zero velocity of the particles in the core of the domain) to red (maximum velocity of the particles at the boundaries)

The preparation of the system followed a standard threestep procedure [17, 22]: (i) frictionless spherical particles are randomly generated in the $3 \mathrm{D}$ box with low volume fraction and random velocities, such that they have sufficient space and time to exchange places and to random- ize themselves; (ii) the inter-particle friction coefficient is activated to a given value $\mu_{0}$ and the granular material is isotropically compressed to the target volume fraction $v$; (iii) the particles are allowed to relax and dissipate their kinetic energy before either a further preparation or the actual element test is initiation.

At the end of the three stages, a static initial granular sample of volume fraction $v$ has been generated, with a certain coordination number $C_{0}$ and a defined isotropic pressure $p_{0}$. By performing steps (ii) and (iii) with different values of the inter-particle friction leads to different isotropic samples (i.e, different values of $C_{0}$ and $p_{0}$ ). For all the target volume fraction considered $(0.59,0.60$ and 0.62$)$, we have performed step (ii) and (iii) by imposing $\mu_{0}=0$ in order to attain initial fluid-like configurations $\left(p_{0}=0 \mathrm{~Pa}\right.$ and $\left.C_{0}=0\right)$. Finally, the inter-particle friction $\mu=0.3$ is assigned, and the boundaries velocity are switched on with global shear rate $\hat{\dot{\gamma}}$, constant in time.

Here and in the following, the variables are made dimensionless using the particle diameter $d$, density $\rho_{p}$ and stiffness $k_{n}$. Then, the scaled time $t$, pressure $p$, shear stress $s$ and shear rate $\dot{\gamma}$ are given, respectively, as: $t \sqrt{k_{n} /\left(\rho_{p} d^{3}\right)}$, $p d / k_{n}, s d / k_{n}$ and $\dot{\gamma} \sqrt{\rho_{p} d^{3} / k_{n}}$.

\section{Results}

The evolution of the dimensionless pressure $p d / k_{n}$ and coordination number $C$ with the accumulated shear strain $\gamma=\hat{\dot{\gamma}} t$ are illustrated in figure 2(a) and (b), respectively.

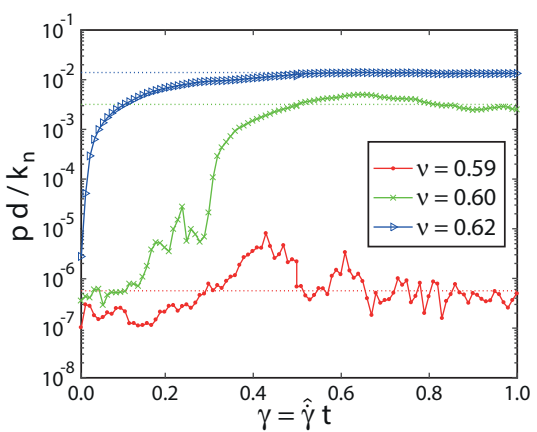

(a)

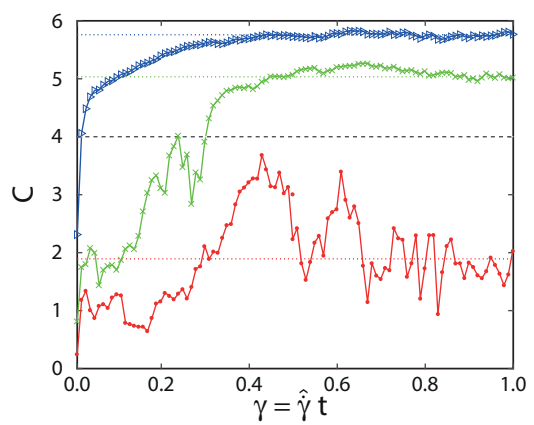

(b)

Figure 2. Evolution of (a) scaled pressure and (b) coordination number for different values of the volume fraction. Steady state values are denoted with dotted lines; the dashed line represents the critical coordination number 
In all the systems, as was already mentioned, both the initial pressure $p_{0}$ and the coordination number $C_{0}$ are zero. At $t=0(\gamma=0)$, a dimensionless global shear rate $\hat{\gamma} \sqrt{\rho_{p} d^{3} / k_{n}}=3.16 \cdot 10^{-5}$ is instantly applied. Pressure and coordination number monotonically increase with time until the steady state is reached, by following similar patterns, as was previously observed by Sun and Sundaresan [21]. The dotted lines in figure 2(a) and (b) represent the final steady state values of $p$ and $C$, obtained by averaging in time over at least 1000 time steps. For $v=0.59$, the steady state is reached in a very short time interval, with large fluctuations for both $p$ and $C$. We take this as a signal that the system is fluid during the simulation; indeed, the coordination number is always less than the critical value 4 (dashed line in figure 2b). In the case $v=0.62$, the system turns rapidly solid, with small fluctuations in both $p$ and $C$, with the former one increasing by several orders of magnitude.

Finally, in the case $v=0.60$ the behaviour of $C$ and $p$ is initially very similar to the fluid case with large oscillations and significant increase in the coordination number. Then, the granular material experiences a fluid-solid transition, and the fluctuations dramatically decrease. In this second stage, the pressure increases of almost 4 orders of magnitude.

The three cases of volume fraction investigated show how much the micro-structure of the system influences the time evolution of the variables. Starting from fluid conditions, that is in absence of force chains, systems at less than critical volume fraction $(v=0.59)$ do not require a long transient regime to reach the steady state, since no persistent chains of contacts among particles form during shear. Although the particles are quite largely spaced and interact by mean of collisions, the coordination number is never zero due to the presence of clusters of particles which do not span the entire domain. The continuous destruction and re-building of clusters, together with the free motion of single particles, gives rise to the observed large fluctuations in both coordination number and pressure. At volume fraction much larger than the critical $(v=0.62)$, the system quickly solidifies during shear. The chains of contacts that span the entire domain require time to develop within the sample and, as a consequence, a clear transient regime is shown in terms of both $C$ and $p$. Once a contact network has been developed, the very small fluctuations of $C$ and $p$ are due to micro-structural rearrangements during shear. The larger the volume fraction, the smaller the fluctuations, since particles are more compacted and cannot easily abandon the own force chain. The transition from fluid to solid is evident in the case $v=0.60$, where the volume fraction is very close to the critical value, and the particles interact through both force chains and collisions. The critical value $C=4$ distinguishes the fluid-like (large fluctuations) from the solid -like (small fluctuations) behaviour (figure 2b).

Finally, the evolution of the stress ratio $s / p$ with the shear strain $\gamma$ is depicted in figure 3(a). Analogously to the other variables, smaller volume fractions are associated with larger oscillations of $s / p$. However, the three

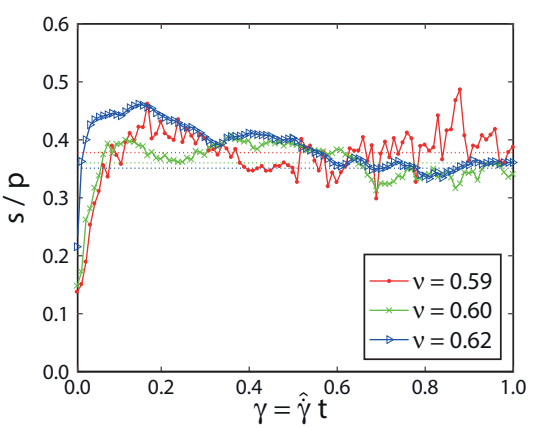

(a)

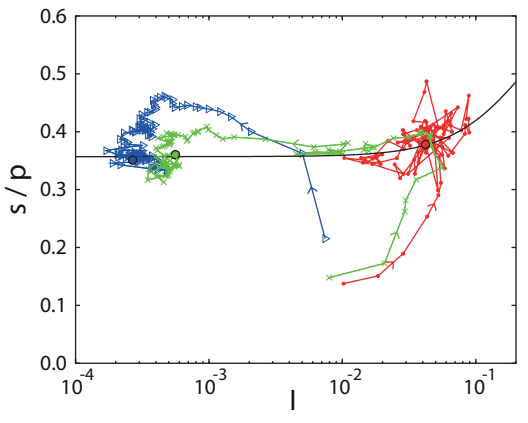

(b)

Figure 3. Stress ratio $s / p$ plotted against (a) strain and (b) inertial number $I$ for different values of the volume fraction. Steady state values of $s / p$ are denoted with dotted line in (a) and filled big circles in (b). The solid line in (b) represents Equation (1) and arrows indicate the direction of increasing $\gamma$

patterns are pretty similar, with an increase at the initial stages, until $\gamma=0.1 \div 0.2$, and then a slight softening to the final steady value.

It is also interesting to plot the stress ratio data measured in the transient against the inertial number: $I=\dot{\gamma} d \sqrt{\rho_{p} / p}$, introduced in Ref. [23-25]. In the case of steady, homogeneous shear flows of rigid particles, the French research group GDR-MiDi [23] has introduced a constitutive relation describing a wide variety of steady flows, at various strain rates. Chialvo et al. [6] have slightly revised that equation, providing a better fit of steady, simple shearing, especially at large $I$ :

$$
\frac{s}{p}=\eta_{0}+\frac{\alpha_{1}}{\left(I_{0} / I\right)^{\beta_{1}}+1}
$$

with $\alpha_{1}=0.667, I_{0}=0.25, \beta_{0}=1.5$ and $\eta_{0}=0.357$ for $\mu=0.3$. In figure 3 (b) our unsteady numerical data (lines with symbols) are compared with Equation (1) (black solid line). During the transient phase, coordination number, pressure and shear stress are homogeneous in the domain, at each time step, whereas, as it was previously observed, the velocity profiles are initially not linear (for $\gamma<0.05$ ) as, instead, is in the case of simple shear flows. This is due to instantaneous application of a relative velocity between the boundaries not immediately affecting the entire granular material. In order to disregard non local effects in this first time periods, we consider the bulk shear rate, averaged over the central region, discarding the regions in proximity of the boundaries. Nevertheless, the 
time interval during which the non-homogeneity is dissipated is very short, $0<\gamma<0.05$, for any volume fraction considered here, and for $\gamma>0.05, \dot{\gamma}$ is homogeneous in space and almost constant in time, equal to the imposed global shear rate $\hat{\dot{\gamma}}$. In figure $3(\mathrm{~b})$, the final steady state values of the three simulations, represented with big filled circles, belong to the curve defined by Equation (1). For $v=0.59$, data taken at different times in the transient phase are characterized by large inertial number $10^{-2}<I<10^{-1}$. Except a few data which lie below the steady state curve, corresponding to the first time steps of the simulation $(0<\gamma<0.1)$, the numerical measurements seem to follow the curve pretty well. The outliers are due to large fluctuations characterizing fluid-like systems, as was previously observed for all the variables. On the other hand, for $v=0.62$, differently from what observed in Ref. [26] for constant pressure simple shearing, Equation (1), nor any linearization of it, cannot predict the behaviour of solid-like, constant volume simple shear flows in the transient regime. The transition from fluid to solid conditions observed for $v=0.60$ in terms of $C$, is evident also in figure 3(b). After a few time steps $(0<\gamma<0.1)$ the data reach the steady state curve at $I \approx 5 \cdot 10^{-2}$, then follow it while $I$ decreases till $I \approx 5 \cdot 10^{-3}$. This behaviour is similar to the fluid case $v=0.59$, with less fluctuations. After that, the data detach from the curve and move above it, denoting the phase transition. Finally, the steady state is attained and just small fluctuations are observed.

\section{Conclusions}

The fluid-solid transition in granular materials has been investigated by performing discrete numerical simulations of unsteady, homogeneous, shear flows. The fluid-like behaviour has been shown to be characterized by large fluctuations in stresses and coordination number. Conversely, the fluctuations are much smaller when a contact network spanning the entire domain develops in the granular material (solid-like behaviour). The fluid-solid transition is characterized by a critical value of the coordination number, independent of the volume fraction. Finally, we have shown that, contrary to what has been observed for unsteady, homogeneous shearing at constant pressure, the relation between the stress ratio and the inertial number, valid for homogeneous steady flows, cannot reproduce the behaviour of unsteady homogeneous shearing at constant volume.

\section{Acknowledgements}

The authors express their special thanks to Professor Stefan Luding for valuable scientific discussions. Dalila
Vescovi is supported by a fellowship from Fondazione Fratelli Confalonieri.

\section{References}

[1] L. Silbert, Soft Matter 6, 2918 (2010)

[2] R. Bagnold, Proc. Royal Society A 255, 49 (1954)

[3] S. Luding, Nature Phys. 12, 531 (2016)

[4] P.C. Johnson, R. Jackson, J. Fluid Mech. 176, 67 (1987)

[5] S.B. Savage, J. Fluid Mech. 377, 1 (1998)

[6] S. Chialvo, J. Sun, S. Sundaresan, Phys. Rev. E 85, 021305 (2012)

[7] D. Vescovi, C. di Prisco, D. Berzi, Int. J. Numer. Anal. Methods Geomech. 37, 2937 (2013)

[8] D. Berzi, J. Jenkins, Soft Matter 11, 4799 (2015)

[9] D. Vescovi, S. Luding, Soft Matter 12, 8616 (2016)

[10] S. Luding, S. McNamara, Granular Matter 1, 113 (1998)

[11] S. Luding, Eur. J. Environ. Civil Eng. 12, 785 (2008)

[12] A. Thornton, T. Weinhart, S. Luding, O. Bokhove, Int. J. Modern Phys. C 23, 1240014 (2012)

[13] T. Weinhart, A. Thornton, S. Luding, O. Bokhove, Granular Matter 14, 289 (2012)

[14] A. Lees, S.F. Edwards, J. Phys. C: Solid State Phys. 5, 1921 (1972)

[15] D. Berzi, D. Vescovi, Phys. Fluids 27, 013302 (2015)

[16] V. Ogarko, S. Luding, Soft Matter 9, 9530 (2013)

[17] N. Kumar, O.I. Imole, V. Magnanimo, S. Luding, Particuology 12, 64 (2014)

[18] S. Ji, H. Shen, Chin. Sci. Bull. 51, 646 (2006)

[19] F. Göncü, O. Duran, S. Luding, Jamming in frictionless packings of spheres: determination of the critical volume fraction, in Powders and Grains 2009, AIP Conference Proc. (M. Nakagawa and S. Luding (Eds.), 2009), Vol. 1145, pp. 531-534

[20] F. Göncü, S. Luding, From "soft" particle simulations to macroscopic constitutive relations, in PARTEC Proc. (Nurnberg Messe GmbH, 2010)

[21] J. Sun, S. Sundaresan, J. Fluid Mech. 682, 590 (2011)

[22] O. Imole, N. Kumar, V. Magnanimo, S. Luding, KONA 30, 84 (2013)

[23] GDR-MiDi, Eur. Phys. J. E 14, 341 (2004)

[24] F. da Cruz, S. Emam, M. Prochnow, J. Roux, F. Chevoir, Phys. Rev. E 72, 021309 (2005)

[25] P. Jop, Y. Forterre, O. Pouliquen, Nature 441, 727 (2006)

[26] A. Ries, L.B.D. Wolf, Comp. Part. Mech. 3, 303 (2016) 